\section{Curbs on research dropped from US medical privacy bill}

[WASHINGTON] A Republican senator has introduced in the US Congress a medical privacy bill that essentially preserves existing procedures for researchers seeking access to identifiable patient information. This is in contrast to a draft of the bill that would have imposed heavy restrictions on researchers and industry (see Nature 392, 6; 1998).

The new version of the bill, from Senator James Jeffords, lacks any requirement for increased scrutiny of Institutional Review Board (IRB) waivers of informed consent. Such waivers are currently allowed for researchers seeking access to identifiable records in cases where it would be impractical to carry out the research if informed consent had to be obtained.

Nor does the Health Care Personal Information Nondisclosure Bill eliminate as the draft did - 'expedited review' for such projects, a procedure under which IRBs may designate a member to give prompt approval.

The bill has also dropped an extension, to the private sector, of protection rules for human subjects, including informed consent for use of identifiable records, that now apply only to federally funded scientists. Industry had said the requirement would be crippling.

"We have worked hard with researchers to make sure we have provided them with the tools necessary" to their work, Jeffords said. "I believe that we have accommodated [their earlier] problems."

Meredith Wadman

\title{
CERN told to start technical thinking for next collider
}

[MUNICH] The European Laboratory for Particle Physics (CERN) in Geneva, Switzerland, has been urged to begin technical design work for the successor to its Large Hadron Collider (LHC), now being built and due to come into operation in 2005.

The SFr 2.6 billion (US $\$ 1.7$ billion) LHC stretches the limits of accelerator technology, and has an expected lifetime of up to 20 years. Its high energy of $14 \mathrm{TeV}$ should allow it to detect the Higgs boson and supersymmetry. An internal report points out that its successor would need to operate at even higher energies. This would require the development of more powerful accelerating systems, or longer tunnels - both very expensive options.

The report was commissioned last summer by CERN's director general, Christopher Llewellyn Smith, and prepared by a small group of CERN particle physicists. It has now been presented to CERN's science programme committee. It suggests that future design work should focus on the development of cost-effective high-field magnets.

It identifies three possibilities for future accelerator concepts. The most studied, in the United States and Japan as well as at CERN, is a $5 \mathrm{TeV}$ electron collider. A second option, a muon collider, is particularly challenging technically because of the rapid rate of decay of muons.
A third option would be a Future Large Hadron Collider (FLHC), a design concept for which has already been developed in the United States (see Nature 385, 471; 1997). This would use a large-circumference, narrow tunnel, built with technology used for laying sewerage pipes. The narrow bore of the tunnel would require the ring to be controlled robotically, rather than by technicians. An electron-positron collider could conceivably share the same tunnel, adding to the possible scientific payoff.

The report says that it is important for CERN to study these concepts now because the technologies will take decades to develop. It will also be many years before particle physicists know what questions remain to be answered after the LHC and other relatively high-energy colliders now being planned have yielded their secrets, and which new type of collider could best answer them.

Tunnel requirements would influence the decision. CERN could conceivably build a tunnel for a muon accelerator at its site, as the characteristics of muons mean that it would be relatively compact. A $30 \mathrm{~km}$ tunnel suitable for a linear electron collider could be located along the nearby Jura mountain range. But the report admits that CERN might find it hard to find a site for a tunnel to meet the requirements of an FLHC, which would need to be at least $120 \mathrm{~km}$ in circumference.

Alison Abbott

\section{Collaboration is the name of the game for Europe's scientists}

[MUNICH] Cooperation between scientists in different European Union (EU) countries has increased significantly over the past decade, according to statistics released by the European Commission last week.

In the ten years from 1985, the funding of research and development in European collaborative programmes rose from 6 to 16 per cent of total spending. That includes the commission's Framework programmes, Eureka and the European-level laboratories.

Framework programmes are designed to stimulate cooperation between researchers in different countries and between industry - particularly small and medium-sized enterprises - and academics. The report says that more than
200,000 such links were established between 1990 and 1996, and that 90 per cent of these were international.

Overall, the statistics show that Europe's international research standing has changed little. The EU invests less in science and technology than its major competitors, the United States and Japan, as a percentage of gross domestic product (1.8, compared with 2.5 and 2.8 respectively). And the number of researchers in Europe is much lower -5 per 1,000 of population. Scientific output, in terms of publications, remains equal to that of the United States, and above that of Japan.

European-level funding is becoming increasingly important for research. Although the proportion of national public

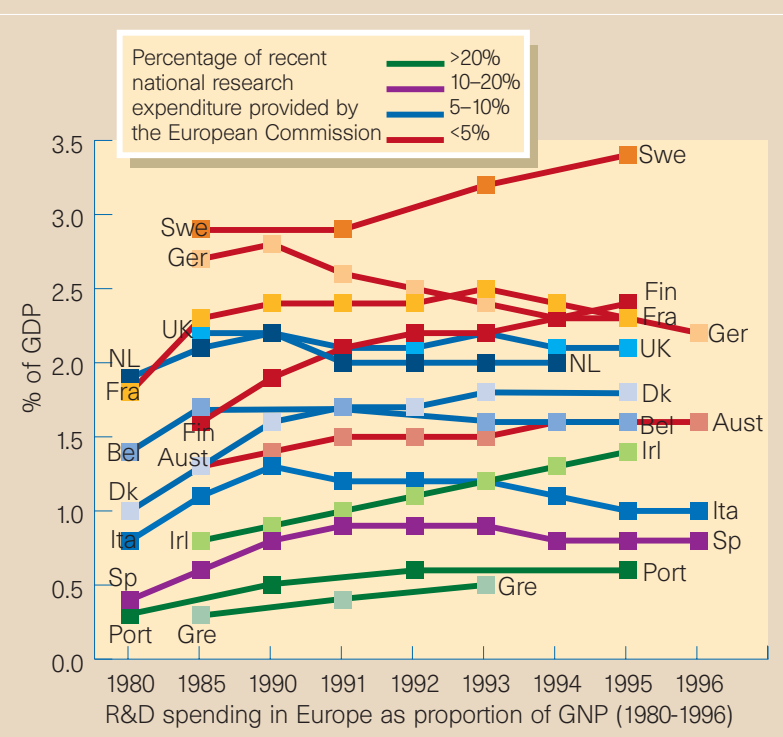

expenditure on research and development fell from an average of 3.5 per cent in 1985 to 2.6 per cent in 1995, research's share of the commission's general budget rose from 2.1 to 5.1 per cent. A.A. 